\title{
What can we learn about gravitational wave physics with an elastic spherical antenna?
}

\author{
J. Alberto Lobo \\ Departament de Física Fonamental, Universitat de Barcelona, Barcelona, Spain
}

(Received 24 January 1995)

\begin{abstract}
A general formalism is set up to analyze the response of an arbitrary solid elastic body to an arbitrary metric gravitational wave (GW) perturbation, which fully displays the details of the interaction antenna wave. The formalism is applied to the spherical detector, whose sensitivity parameters are thereby scrutinized. A multimode transfer function is defined to study the amplitude sensitivity, and absorption cross sections are calculated for a general metric theory of GW physics. Their scaling properties are shown to be independent of the underlying theory, with interesting consequences for future detector design. The GW incidence direction deconvolution problem is also discussed, always within the context of a general metric theory of the gravitational field.
\end{abstract}

PACS number(s): $04.80 . \mathrm{Nn}, 95.55 . \mathrm{Ym}$

\section{INTRODUCTION}

The idea of building ultracryogenic spherical gravitational wave (GW) antennae seems to be progressively winning adherents, even despite the technological difficulties of various kinds posed by a project such as this, which every expert acknowledges. Confidence in its feasibility stems from many years of experience: Groups at Stanford, Louisiana State University, Roma and Legnaro (Italy), and Perth (Western Australia) have constructed and operated, at different levels, cryogenic cylindrical bars of the Weber type [1]. In particular, a long term strain sensitivity $h=6 \times 10^{-19}$ for millisecond bursts has been reported from the Rome Explorer [2] bar. The new generation ultracryogenic cylinder Nautilus, of the Frascati group [3], is currently beginning operation [4], with an expected sensitivity nearly an order of magnitude better than the above.

Spherical antennae are considered by many to be the natural next step in the development of resonant GW detectors [5-10]. The reasons for this new trend essentially derive from the improved sensitivity of a sphere, which can be nearly an order of magnitude better than a cylinder having the same resonance frequency (see below and [10]), and from its multimode capabilities, first recognized by Forward [5] and further elaborated in $[7,8]$.

Although some of the most relevant aspects of detector sensitivity have already received attention in the literature, it appears to me that a sufficiently general and flexible analysis of the detector-GW interaction has not been satisfactorily developed to date. This theoretical shortage has a number of practical negative consequences, too. Traditional analysis, to mention but an example, is almost invariably restricted to general relativity or scalartensor theories of gravity; while it may be argued that this is already very general, any such argument is, as a matter of fact, understating the potentialities actually offered by a spherical GW antenna to help decide for or against any one specific theory of the gravitational field on the basis of experimental observation.
I thus propose to develop in this paper a full fledged mathematical formalism which will enable analysis of the antenna's response to a completely general GW, i.e., making no a priori assumptions about which is the correct theory underlying GW physics (other than, indeed, that it is a metric theory), and also making no assumptions about detector shape, structure, or boundary conditions. Considering things in such a generality is not only "theoretically nice," it also brings about new results and a better understanding of older ones. For example, it will be proved that the sphere is the most efficient GW elastic detector shape, and that higher mode absorption cross sections scale independently of $G W$ physics. I will also discuss the direction of the incidence deconvolution problem in the context of a general metric theory of gravity.

The paper is organized as follows. Section II is devoted to the development of the general mathematical framework, leading to a formula in which an elastic solid's response is related to the action of an arbitrary metric GW impinging on it. In Sec. III the general equations are applied to the homogeneous spherical body, and a discussion of the deconvolution problem is presented as well. Section IV contains the description of the sphere's sensitivity parameters, specifically leading to the concept of multimode, or vector, transfer function, and to an analysis of the absorption cross section presented by this detector to a passing by GW. Conclusions and prospects are summarized in Sec. V, and two appendixes are added which include mathematical derivations.

\section{GENERAL MATHEMATICAL FRAMEWORK}

In the mathematical model, I shall be assuming that the antenna is a solid elastic body which responds to GW perturbations according to the equations of classical nonrelativistic linear elasticity theory [11]. This is fully justified since, as stressed above, GW-induced dis- 
placements will be very small indeed, and the speed of such displacements much smaller than that of light for any forseeable frequencies. Although our primary interest is a spherical antenna, the considerations which follow in the remainder of this section have general validity for arbitrarily shaped isotropic elastic solids.

Let $\mathbf{u}(\mathbf{x}, t)$ be the displacement vector of the infinitesimal mass element sitting at point $\mathbf{x}$ relative to the solid's center of mass in its unperturbed state, whose density distribution in that state is $\rho(\mathbf{x})$. Let $\lambda$ and $\mu$ be the material's elastic Lamé coefficients. If a volume force density $\mathbf{f}(\mathbf{x}, t)$ acts on such a solid, the displacement field $\mathbf{u}(\mathbf{x}, t)$ is the solution to the system of partial differential equations [11]:

$$
\rho \frac{\partial^{2} \mathbf{u}}{\partial t^{2}}-\mu \nabla^{2} \mathbf{u}-(\lambda+\mu) \nabla(\nabla \cdot \mathbf{u})=\mathbf{f}(\mathbf{x}, t),
$$

with the appropriate initial and boundary conditions. A summary of notation and general results regarding the solution to that system is briefly outlined in the ensuing subsection, as they are necessary for the subsequent developments in this paper, and also in future work.

\section{A. Separable driving force}

For reasons which will become clear later on, we shall only be interested in driving forces of the separable type,

$$
\mathbf{f}(\mathbf{x}, t)=\mathbf{f}(\mathbf{x}) g(t)
$$

or, indeed, linear combinations thereof. The solution to (2.1) does not require us to specify the precise boundary conditions on $\mathbf{u}(\mathbf{x}, t)$ at this stage, but we need to set the initial conditions. We adopt

$$
\mathbf{u}(\mathbf{x}, 0)=\dot{\mathbf{u}}(\mathbf{x}, 0)=0,
$$

where an overdot denotes $\partial / \partial t$, implying that the antenna is at complete rest before observation begins at $t=0$. The structure of the force field (2.2) is such that the displacements $\mathbf{u}(\mathbf{x}, t)$ can be expressed by means of a Green function integral of the form

$$
\mathbf{u}(\mathbf{x}, t)=\int_{0}^{\infty} \mathbf{S}\left(\mathbf{x} ; t-t^{\prime}\right) g\left(t^{\prime}\right) d t^{\prime} .
$$

The deductive procedure whereby $\mathbf{S}\left(\mathbf{x} ; t-t^{\prime}\right)$ is calculated can be found in many standard textbooks - see, e.g., [12]. The result is

$$
\mathbf{S}(\mathbf{x} ; t)= \begin{cases}0 & \text { if } t \leq 0, \\ \sum_{N} \frac{f_{N}}{\omega_{N}} \mathbf{u}_{N}(\mathbf{x}) \sin \omega_{N} t & \text { if } t \geq 0,\end{cases}
$$

where

$$
f_{N} \equiv \frac{1}{M} \int_{\text {solid }} \mathbf{u}_{N}^{*}(\mathbf{x}) \cdot \mathbf{f}(\mathbf{x}) d^{3} x
$$

and $\mathbf{u}_{N}(\mathbf{x})$ are the normalized eigensolutions to

$$
\mu \nabla^{2} \mathbf{u}_{N}+(\lambda+\mu) \nabla\left(\nabla \cdot \mathbf{u}_{N}\right)=-\omega_{N}^{2} \rho \mathbf{u}_{N}
$$

with suitable boundary conditions. Here $N$ represents an index, or set of indices, labeling the eigenmode of frequency $\omega_{N}$. The normalization condition is (arbitrarily) chosen so that

$$
\int_{\text {solid }} \mathbf{u}_{N^{\prime}}^{*}(\mathbf{x}) \cdot \mathbf{u}_{N}(\mathbf{x}) \rho(\mathbf{x}) d^{3} x=M \delta_{N^{\prime} N}
$$

where $M$ is the total mass of the solid, and the asterisk denotes complex conjugation. Replacing now (2.5) into (2.4) we can write the solution to our problem as a series expansion:

$$
\mathbf{u}(\mathbf{x}, t)=\sum_{N} \frac{f_{N}}{\omega_{N}} \mathbf{u}_{N}(\mathbf{x}) g_{N}(t)
$$

where

$$
g_{N}(t) \equiv \int_{0}^{t} g\left(t^{\prime}\right) \sin \omega_{N}\left(t-t^{\prime}\right) d t^{\prime}
$$

Equation (2.9) is the formal solution to our problem; it has the standard form of an orthogonal expansion and is valid for any solid driven by a separable force such as (2.2) and any boundary conditions. It is therefore completely general, given that type of force.

Before we go on, it is perhaps interesting to quote a simple but useful example. It is the case of a solid hit by a hammer blow, i.e., receiving a sudden stroke at a point on its surface. An examination of the response of a GW antenna to such perturbation is being used for correct tuning and monitoring of the device [13]. If the driving force density is represented by the simple model

$$
\mathbf{f}^{(\mathbf{h b})}(\mathbf{x}, t)=\mathbf{f}_{0} \delta^{(3)}\left(\mathbf{x}-\mathbf{x}_{0}\right) \delta(t),
$$

where $\mathbf{x}_{0}$ is the surface point hit, and $\mathbf{f}_{0}$ is a constant vector, then the system's response is immediately seen to be

$$
\mathbf{u}^{(\mathrm{hb})}(\mathbf{x}, t)=\sum_{N} \frac{f_{N}^{0}}{\omega_{N}} \mathbf{u}_{N}(\mathbf{x}) \sin \omega_{N} t
$$

with $f_{N}^{0}=M^{-1} \mathbf{f}_{0} \cdot \mathbf{u}_{N}^{*}\left(\mathbf{x}_{0}\right)$. A hammer blow thus excites all the solid's normal modes, except those perpendicular to $\mathbf{f}_{0}$, with amplitudes which are inversely proportional to the mode's frequency. This is seen to be a rather general result in the theory of sound waves in isotropic elastic solids.

\section{B. GW tidal forces}

An incoming GW manifests itself as a tidal force density; in the long-wavelength linear approximation [14] it only depends on the "electric" components of the Riemann tensor:

$$
f_{i}(\mathbf{x}, t)=\rho c^{2} R_{0 i 0 j}(t) x_{j}
$$


where $c$ is the speed of light, and a sum over the repeated index $j$ is understood. In (2.13) tidal forces are referred to the antenna's center of mass, and thus $\mathbf{x}$ is a vector originating there. Note that I have omitted any dependence of $R_{0 i 0 j}$ on spatial coordinates, since it only needs to be evaluated at the solid's center. The Riemann tensor is only required to first order at this stage [15]:

$$
R_{0 i 0 j}=\frac{1}{2}\left(h_{i j, 00}-h_{0 i, 0 j}-h_{0 j, 0 i}+h_{00, i j}\right)
$$

where $h_{\mu \nu}$ are the perturbations to flat geometry, ${ }^{1}$ always at the center of mass of the detector.

The form (2.13) is seen to be a sum of three terms such as (2.2), but this three term "straightforward" splitting is not the most convenient, due to the lack of invariance and symmetry. A better choice is now outlined.

An arbitrary symmetric tensor $\mathcal{S}_{i j}$ admits the decomposition

$$
\mathcal{S}_{i j}(t)=\mathcal{S}^{(S)}(t) E_{i j}^{(S)}+\sum_{m=-2}^{2} \mathcal{S}^{(m)}(t) E_{i j}^{(m)}
$$

where $E_{i j}^{(m)}$ are five linearly independent symmetric and traceless tensors, and $E_{i j}^{(S)}$ is a multiple of the unit tensor $\delta_{i j} \cdot \mathcal{S}^{(S)}(t)$ and $\mathcal{S}^{(m)}(t)$ are uniquely defined functions, whose explicit form depends on the particular representation of the $E$ matrices chosen. A convenient one is

$$
\begin{aligned}
& E_{i j}^{(S)}=\left(\frac{1}{4 \pi}\right)^{\frac{1}{2}}\left(\begin{array}{lll}
1 & 0 & 0 \\
0 & 1 & 0 \\
0 & 0 & 1
\end{array}\right) \\
& E_{i j}^{(0)}=\left(\frac{5}{16 \pi}\right)^{\frac{1}{2}}\left(\begin{array}{ccc}
-1 & 0 & 0 \\
0 & -1 & 0 \\
0 & 0 & 2
\end{array}\right), \quad E_{i j}^{( \pm 1)}=\left(\frac{15}{32 \pi}\right)^{\frac{1}{2}}\left(\begin{array}{ccc}
0 & 0 & \mp 1 \\
0 & 0 & -i \\
\mp 1 & -i & 0
\end{array}\right), \quad E_{i j}^{( \pm 2)}=\left(\begin{array}{cc}
\frac{15}{32 \pi}
\end{array}\right)^{\frac{1}{2}}\left(\begin{array}{ccc}
1 & \pm i & 0 \\
\pm i & -1 & 0 \\
0 & 0 & 0
\end{array}\right)
\end{aligned}
$$

The excellence of this representation stems from its ability to display the spin features of the driving terms in (2.13). Such features are characterized by the relations

$$
E_{i j}^{(S)} n_{i} n_{j}=Y_{00}(\theta, \varphi) \quad, \quad E_{i j}^{(m)} n_{i} n_{j}=Y_{2 m}(\theta, \varphi),
$$

where $\mathbf{n} \equiv \mathbf{x} /|\mathbf{x}|$ is the radial unit vector, and $Y_{l m}(\theta, \varphi)$ are spherical harmonics [16]. Details about the above $E$ matrices are given in Appendix A. In particular, the orthogonality relations (A6) can be used to invert (2.15):

$$
\mathcal{S}^{(S)}(t)=\frac{4 \pi}{3} E_{i j}^{(S)} \mathcal{S}_{i j}(t)
$$

$$
\mathcal{S}^{(m)}(t)=\frac{8 \pi}{15} E_{i j}^{*(m)} \mathcal{S}_{i j}(t), \quad m=-2, \ldots, 2,
$$

where an asterisk denotes complex conjugation. Note that $\mathcal{S}^{(S)}(t)=\sqrt{4 \pi} \mathcal{S}(t) / 3$, where $\mathcal{S}(t) \equiv \delta_{i j} \mathcal{S}_{i j}(t)$ is the tensor's trace.

We now take advantage of (2.15) to express the GW tidal force (2.13) as a sum of split terms such as (2.2):

$$
\mathbf{f}(\mathbf{x}, t)=\mathbf{f}^{(S)}(\mathbf{x}) g^{(S)}(t)+\sum_{m=-2}^{2} \mathbf{f}^{(m)}(\mathbf{x}) g^{(m)}(t)
$$

with

$$
\begin{aligned}
& f_{i}^{(S)}(\mathbf{x})=\rho E_{i j}^{(S)} x_{j}, g^{(S)}(t)=\frac{4 \pi}{3} E_{i j}^{*(S)} R_{0 i 0 j}(t) c^{2}, \\
& f_{i}^{(m)}(\mathbf{x})=\rho E_{i j}^{(m)} x_{j} \quad, \quad g^{(m)}(t)=\frac{8 \pi}{15} E_{i j}^{*(m)} R_{0 i 0 j}(t) c^{2} \quad(m=-2, \ldots, 2) .
\end{aligned}
$$

Straightforward application of (2.9) yields the formal solution of the antenna response to a GW perturbation:

\footnotetext{
${ }^{1}$ Throughout this paper, greek indices $(\mu, \nu, \ldots)$ will run through space-time values $0,1,2,3 ;$ latin indices $(i, j, \ldots)$ will run through space values $1,2,3$ only.
} 


$$
\mathbf{u}(\mathbf{x}, t)=\sum_{N} \omega_{N}^{-1} \mathbf{u}_{N}(\mathbf{x})\left[f_{N}^{(S)} g_{N}^{(S)}(t)+\sum_{m=-2}^{2} f_{N}^{(m)} g_{N}^{(m)}(t)\right]
$$

with the notation of (2.6) and (2.10) applied mutatis $m u$ tandi to the terms in (2.20).

Equation (2.21) gives the response of an arbitrary elastic solid to an incoming weak $\mathrm{GW}$, independently of the underlying gravity theory, be it general relativity (GR) or indeed any other metric theory of the gravitational interaction. It is also valid for any antenna shape and any boundary conditions, thus giving the formalism, in particular, the capability of being used to study the response of a detector which is suspended by means of a mechanical device in the laboratory site - a situation of much practical importance. It is therefore very general.

Equation (2.21) also tells us that that only monopole and quadrupole detector modes can possibly be excited by a metric GW. The nice thing about (2.21) is that it fully displays the monopole-quadrupole structure of the solution to our fundamental differential equations.

In a nonsymmetric body, all (or nearly all) the modes have monopole and quadrupole moments, and (2.21) precisely shows how much each of them contributes to the detector's response. A homogeneous spherical antenna, which is very symmetric, has a set of vibrational eigenmodes which are particularly well matched to the form (2.21): It only possesses one series of monopole modes and one (fivefold degenerate) series of quadrupole modes - see the next section and Appendix B for details. The existence of so few modes which couple to GW's means that all the absorbed incoming radiation energy will be distributed amongst those few modes only, thereby making the sphere the most efficient detector, even from the sensitivity point of view. The higher-energy cross section per unit mass reported for spheres on the basis of GR [10], for example, finds here its qualitative explanation. The generality of (2.21), on the other hand, means that this excellence of the spherical detector is there independently of which is the correct $G W$ theory.

Before going further, let me mention another potentially useful application of the formalism so far. Cylindrical antennas, for instance, are usually studied in the thin rod approximation; although this is generally quite satisfactory, Eq. (2.21) offers the possibility of eventually considering corrections to such a simplifying hypothesis by use of more realistic eigenfunctions, such as those given in $[17,18]$. Recent new proposals for stumpy cylinder arrays [19] may well benefit from the above approach, too.

\section{SPHERICAL ANTENNA}

To explore the consequences of (2.21) in a particular case, the mode amplitudes $\mathbf{u}_{N}(\mathbf{x})$ and frequencies $\omega_{N}$ must be specified. From now on I will focus on a homogeneous sphere whose surface is free of tractions and/or tensions; the latter happens to be quite a good approx- imation, even if the sphere is suspended in the static gravitational field [20].

The normal modes of the free sphere fall into two families: so-called toroidal, where the sphere only undergoes twistings which keep its shape unchanged throughout the volume, and spheroidal [21], where radial as well as tangential displacements take place. I use the notation

$$
\mathbf{u}_{n l m}^{T}(\mathbf{x}) e^{ \pm i \omega_{n l}^{T} t}, \quad \mathbf{u}_{n l m}^{P}(\mathbf{x}) e^{ \pm i \omega_{n l}^{P} t}
$$

for them, respectively; note that the index $N$ of the previous section is a multiple index $\{n l m\}$ for each family; $l$ and $m$ are the usual multipole indices, and $n$ numbers from 1 to $\infty$ each of the $l$-pole modes. The frequencies happen to be independent of $m$, and so every one mode (3.1) is $(2 l+1)$-fold degenerate. Further details about these eigenmodes are given in Appendix B.

In order to see what (2.21) looks like in this case, integrals of the form (2.6) ought to be evaluated. It is straightforward to prove that they all vanish for the toroidal modes, the spheroidal modes contributing the only nonvanishing terms; after some algebra one finds

$$
\begin{aligned}
f_{n l m}^{(S)} & \equiv \frac{1}{M} \int_{\text {sphere }} \mathbf{u}_{n l m}^{P *}(\mathbf{x}) \cdot \mathbf{f}^{(S)}(\mathbf{x}) d^{3} x=a_{n} \delta_{l 0} \delta_{m 0}, \\
f_{n l m}^{\left(m^{\prime}\right)} & \equiv \frac{1}{M} \int_{\text {sphere }} \mathbf{u}_{n l m}^{P *}(\mathbf{x}) \cdot \mathbf{f}^{\left(m^{\prime}\right)}(\mathbf{x}) d^{3} x=b_{n} \delta_{l 2} \delta_{m^{\prime} m},
\end{aligned}
$$

where

$$
\begin{aligned}
a_{n} & =-\frac{1}{M} \int_{0}^{R} A_{n 0}(r) \rho r^{3} d r \\
b_{n} & =-\frac{1}{M} \int_{0}^{R}\left[A_{n 2}(r)+3 B_{n 2}(r)\right] \rho r^{3} d r .
\end{aligned}
$$

The functions $A_{n l}(r), B_{n l}(r)$ are given in Appendix B, and $R$ is the sphere's radius. To our reassurance, only the monopole and quadrupole sphere modes survive, as seen by the presence of the factors $\delta_{l 0}$ and $\delta_{l 2}$ in (3.2a) and (3.2b), respectively. The final series is thus a relatively simple one, even in spite of its generality: ${ }^{2}$

\footnotetext{
${ }^{2}$ From now on I will drop the label $P$, meaning spheroidal mode, to ease the notation since toroidal modes no longer appear in the formulas.
} 


$$
\mathbf{u}(\mathbf{x}, t)=\sum_{n=1}^{\infty} \frac{a_{n}}{\omega_{n 0}} \mathbf{u}_{n 00}(\mathbf{x}) g_{n 0}^{(S)}(t)+\sum_{n=1}^{\infty} \frac{b_{n}}{\omega_{n 2}}\left[\sum_{m=-2}^{2} \mathbf{u}_{n 2 m}(\mathbf{x}) g_{n 2}^{(m)}(t)\right] \quad(t>0)
$$

where, it is recalled,

$$
g_{n l}^{(S, m)}(t)=\int_{0}^{t} g^{(S, m)}\left(t^{\prime}\right) \sin \omega_{n l}\left(t-t^{\prime}\right) d t^{\prime} \quad(m=-2, \ldots, 2)
$$

Equation (3.4) constitutes the sphere's response to an arbitrary tidal GW perturbation, and will be used to analyze the sensitivity of the spherical detector in the next section. Before doing so, however, a few comments on the antenna's signal deconvolution capabilities, within the context of a completely general metric theory of GW's, are in order.

\section{A. Deconvolution problem}

Let us first of all take the Fourier transform of (3.4):

$$
\mathbf{U}(\mathbf{x}, \dot{\omega}) \equiv \int_{-\infty}^{\infty} \mathbf{u}(\mathbf{x}, t) e^{-i \omega t} d t
$$

This is seen to be

$$
\begin{aligned}
\mathbf{U}(\mathbf{x}, \omega)= & \frac{\pi}{i} \sum_{n=1}^{\infty} \frac{a_{n}}{\omega_{n 0}} \mathbf{u}_{n 00}(\mathbf{x}) G^{(S)}(\omega)\left[\delta\left(\omega-\omega_{n 0}\right)-\delta\left(\omega+\omega_{n 0}\right)\right] \\
& +\frac{\pi}{i} \sum_{n=1}^{\infty} \frac{b_{n}}{\omega_{n 2}}\left[\sum_{m=-2}^{2} \mathbf{u}_{n 2 m}(\mathbf{x}) G^{(m)}(\omega)\right]\left[\delta\left(\omega-\omega_{n 2}\right)-\delta\left(\omega+\omega_{n 2}\right)\right]
\end{aligned}
$$

where $G^{(S)}(\omega)$ and $G^{(m)}(\omega)$ are the Fourier transforms of $g^{(S)}(t)$ and $g^{(m)}(t)$, respectively:

$$
G^{(S, m)}(\omega) \equiv \int_{0}^{\infty} g^{(S, m)}(t) e^{-i \omega t} d t
$$

The $\delta$-function factors are of course idealizations corresponding to infinitely long integration times and infinitely narrow resonance linewidths, but the essentials of the ensuing discussion will not be affected by those idealizations.

If the measuring system were (ideally) sensitive to all frequencies, filters could be applied to examine the antenna's oscillations at each monopole and quadrupole frequency: A single transducer would suffice to reveal $G^{(S)}(\omega)$ around the monopole frequencies $\omega_{n 0}$, while five (placed at suitable positions) would be required to calculate the five degenerate amplitudes $G^{(m)}(\omega)$ around the quadrupole frequencies $\omega_{n 2}$. Once the six functions $G^{(S, m)}(\omega)$ would have thus been determined, inverse Fourier transforms would give us the functions $g^{(S, m)}(t)$, and thereby the six Riemann tensor components $R_{0 i 0 j}(t)$ through inversion of the second equations (2.20), i.e., as an expansion like (2.15), only with $g$ 's instead of $\mathcal{S}$ 's. Deconvolution would then be complete.

Well, not quite. Knowledge of the Riemann tensor in the laboratory frame coordinates is not really sufficient to say the waveform has been completely deconvolved, unless we also know the source position in the sky. There clearly are two possibilities.

(i) The source position is known ahead of time by some other astronomical observation methods. Let me rush to emphasise that, far from trivial or uninteresting, this is a very important case to consider, especially during the first stages of GW astronomy, when any reported GW event will have to be thoroughly checked by all possible means.

If the incidence direction is known, then a rotation must be applied to the just obtained quantities $R_{0 i 0 j}(t)$, which takes the laboratory $z$ axis into coincidence with the incoming wave propagation vector. A classification procedure must thereafter be applied to the sotransformed Riemann tensor in order to see which is the theory (or class of theories) compatible with the actual observations. Such classification procedure has been described in detail in [22].

The spherical antenna is thus seen to have the capability of furnishing the analyst sufficient information to discern amongst different competing theories of $G W$ physics, whenever the wave incidence direction is known prior to detection.

(ii) The source position is not known at detection time. This makes things more complex, since the above rotation between the laboratory and GW frames cannot be performed.

In order to deconvolve the incidence direction in this case, a specific theory of the GW's must be assumed, a given choice being made on the basis of whatever prior information is available or, simply, dictated by the de- 
cision to probe a particular theory. Wagoner and Paik [7] propose a method which is useful both for GR and Brans-Dicke (BD) theory, their idea being simple and elegant at the same time: Since neither of these theories predicts the excitation of the $m= \pm 1$ quadrupole modes of the wave, the source position is determined precisely by the rotation angles which, when applied to the laboratory axes, cause the amplitudes of those antenna modes to vanish; the rotated frame is thereby associated to the GW natural frame.

A generalization of this idea can conceivably be found on the basis of a detailed, and possibly rather casuistic, analysis of the canonical forms of of the Riemann tensor for a list of theories of gravity, along the following line of argument: Any one particular theory will be characterized by certain (homogeneous) canonical relationships among the monopole and quadrupole components of the Riemann tensor, $g^{(S, m)}(t)$, and so enforcement of those relations upon rotation of the laboratory frame axes should enable determination of the rotation angles or, equivalently, of the incoming radiation incidence direction. Scalar-tensor theories, e.g., have $g^{( \pm 1)}(t)=0$ in their canonical forms, hence Wagoner and Paik's proposal for this particular case.

Before any deconvolution procedure is triggered off, however, it is very important to make sure that it will be viable. More precisely, since the transformation from the laboratory to the ultimate canonical frame is going to be linear, invariants must be preserved. This means that, even if the source position is unknown, certain theories will forthrightly be vetoed by the observed $R_{0 i 0 j}(t)$ if their predicted invariants are incompatible with the observed ones. To give but an easy example, if $R_{0 i 0 j}(t)$ is observed to have a non-null trace $R_{0 i 0 i}(t)$, then a veto on GR will be readily served, and therefore no algorithm based on that theory should be applied.

I would like to make a final remark here. Assume a direction deconvolution procedure has been successfully carried through to the end on the basis of certain GW theory, so that the analyst comes up with a pair of numbers $(\theta, \varphi)$ expressing the source's coordinates in the sky. Of course, these numbers will represent the actual source position only if the assumed theory is correct. Now, how do we know it is correct? Strictly speaking, "correctness" of a scientific theory is an asymptotic concept, in the sense that the possibility always remains open that new facts be eventually discovered which contradict the theory, and so reliability of the estimate $(\theta, \varphi)$ of the source position can only be assessed in practice in terms of the consistency between the assumed theory and whatever experimental evidence is available to date, including, indeed, GW measurements themselves. It is thus very important to have a method to verify that the estimate $(\theta, \varphi)$ does not contradict the theory which enabled its very determination.

Such verification is a logical absurdity if only one measurement of position is available; this happens for instance if the recorded signal is a short burst of radiation, and so two antennas are at least necessary to check consistency in that case. The test would proceed as a check that the time delay between reception of the signal at both detectors is consistent with the calculated $(\theta, \varphi),^{3}$ given their relative position and the wave propagation speed predicted by the assumed theory. If, on the other hand, the signal being tracked is a long duration signal, then a single antenna may be sufficient to peform the test by looking at the observed Doppler patterns and checking them against those expected with the given $(\theta, \varphi)$.

The above considerations have been made ignoring noise in the detector and monitor systems. A fundamental constraint introduced by noise is that it makes the antenna bandwidth limited in sensitivity. As a consequence, any deconvolution procedure is deemed to be incomplete or, rather, ambiguous [23], since information about the signal can possibly be retrieved only within a reduced bandwidth, while the rest will be lost. I thus come to a detailed discussion of the sensitivity of the spherical GW antenna in the next section.

\section{SENSITIVITY PARAMETERS}

I will consider successively amplitude and energy sensitivities; the first leads to the concept of transfer function, while the second to that of absorption cross section. I devote separate subsections to analyze each of them in some detail.

\section{A. Transfer function}

A widely used and useful concept in linear system theory is that of transfer function [24]. It is defined as the Fourier transform of the system's impulse response, or as the system's impedance and/or admittance, and can be inferred from the frequency response function (3.7).

We recall from the previous section that the sphere is a multimode device, due to its monopole and fivefold degenerate quadrupole modes. It appears expedient to define a multimode or vector transfer function as a useful construct which encompasses all six different modes into a single conceptual block, according to

$$
\mathbf{U}(\mathbf{x}, \omega)=\sum_{\alpha} \mathbf{Z}^{(\alpha)}(\mathbf{x}, \omega) G^{(\alpha)}(\omega),
$$

where $G^{(\alpha)}(\omega)$ are the six driving terms $G^{(S, m)}(\omega)$ given in (3.8). The transfer function is $\mathbf{Z}^{(\alpha)}(\mathbf{x}, \omega)$, and its "vector" character alluded to above is reflected by the multimode index $\alpha$. Looking at (3.7) it is readily seen that

\footnotetext{
${ }^{3}$ Note that the two detectors will agree on the same $(\theta, \varphi)$, even if the assumed theory is wrong, since the sphere deformations will be the same if caused by the same signal.
} 


$$
\begin{aligned}
& \mathbf{Z}^{(S)}(\mathbf{x}, \omega)=\frac{\pi}{i} \sum_{n=1}^{\infty} \frac{a_{n}}{\omega_{n 0}} \mathbf{u}_{n \mathbf{0 0}}(\mathbf{x})\left[\delta\left(\omega-\omega_{n 0}\right)-\delta\left(\omega+\omega_{n 0}\right)\right], \\
& \mathbf{Z}^{(m)}(\mathbf{x}, \omega)=\frac{\pi}{i} \sum_{n=1}^{\infty} \frac{b_{n}}{\omega_{n 2}} \mathbf{u}_{n 2 m}(\mathbf{x})\left[\delta\left(\omega-\omega_{n 2}\right)-\delta\left(\omega+\omega_{n 2}\right)\right](m=-2, \ldots, 2) .
\end{aligned}
$$

As we observe in these formulas, the sphere's sensitivity to monopole excitations is governed by $a_{n} / \omega_{n 0}$ and to quadrupole ones by $b_{n} / \omega_{n 2}$. Closed expressions happen to exist for $a_{n}$ and $b_{n}$; using the notation of Appendix B, they are

$$
\begin{aligned}
& \frac{a_{n}}{R}=\frac{3 C(n, 0)}{8 \pi} \frac{j_{2}\left(q_{n 0} R\right)}{q_{n 0} R} \\
& \frac{b_{n}}{R}=-\frac{3 C(n, 2)}{8 \pi}\left[\beta_{3}\left(k_{n 2} R\right) \frac{j_{2}\left(q_{n 2} R\right)}{q_{n 2} R}-3 \frac{q_{n 2}}{k_{n 2}} \beta_{1}\left(q_{n 2} R\right) \frac{j_{2}\left(k_{n 2} R\right)}{k_{n 2} R}\right] .
\end{aligned}
$$

Numerical investigation of the behavior of these coefficients shows that they decay asymptotically as $n^{-2}$ :

$$
a_{n}, b_{n} \sim \text { const } \times n^{-2} .
$$

Likewise, it is found that the frequencies $\omega_{n 0}$ and $\omega_{n 2}$ diverge like $n$ for large $n$, so that $\mathbf{Z}^{(\alpha)}(\mathbf{x}, \omega)$ drops as $\omega^{-3}$ for large $\omega$. Figures 1 and 2 display a symbolic plot of $\omega^{3} \mathbf{Z}^{(S)}(\mathbf{x}, \omega)$ and $\omega^{3} \mathbf{Z}^{(m)}(\mathbf{x}, \omega)$, respectively, which illustrates the situation: Monopole modes soon reach the asymptotic regime, while there appear to be three subfamilies of quadrupole modes regularly intertwined; the asymptotic regime for these subfamilies is more irregularly reached. Note also the perfectly regular alternate changes of phase (by $\pi$ radians) in both monopole and each quadrupole family.

The sharp fall in sensitivity of a sphere for higherfrequency modes $\left(n^{-3}\right)$ indicates that only the lowest ones stand a chance of being obervable in an actual

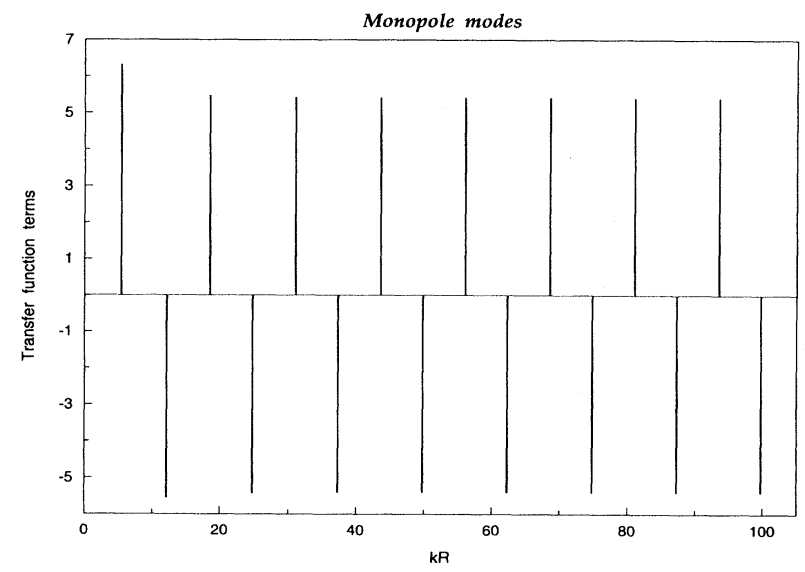

FIG. 1. The scalar component $\mathbf{Z}^{(S)}(\mathbf{x}, \omega)$ of the multimode transfer function, (4.2a). The diagram actually displays $\omega^{3} \mathbf{Z}^{(S)}(\mathbf{x}, \omega)$, and so asymptotic behaviors are better appreciated. It is given in units of $\mu / \rho R$, and a factor $(\pi / i) \mathbf{u}_{n 00}(\mathbf{x})$, the eigenmode amplitude, has been omitted, too. $\delta$-function amplitudes are symbolically taken as 1 . Note that the asymptotic regime, given by Eq. (4.4), is quickly reached.
GW antenna. I report in Table I the numerical values of the relevant parameters for the first few monopole and quadrupole modes. The reason for the last (fourth) columns will become clear later.

\section{B. Absorption cross section}

Let us calculate now the energy of the oscillating sphere. We first define the spectral energy density at frequency $\omega$, which is naturally given by ${ }^{4}$

$$
W(\omega)=\frac{1}{T} \int_{\text {solid }} \frac{1}{2} \omega^{2}|\mathbf{U}(\mathbf{x}, \omega)|^{2} \rho d^{3} x
$$

and can be easily evaluated:

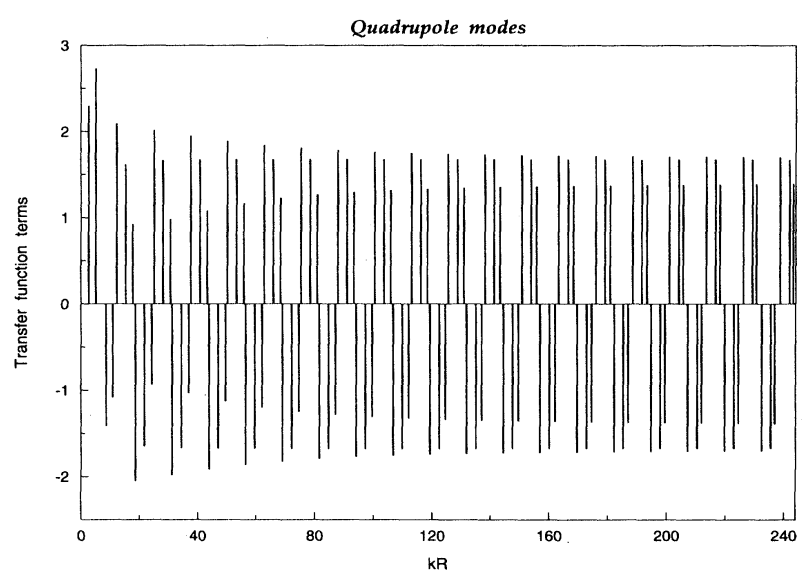

FIG. 2. The quadrupole component $\mathbf{Z}^{(m)}(\mathbf{x}, \omega)$ of the multimode transfer function, $(4.2 \mathrm{~b})$. The same prescriptions of Fig. 6 apply here; the plot is therefore independent of the value of $m$. Note the presence of three subfamilies of peaks; asymptotic regimes are reached with variable speed for these subfamilies, and less rapidly than for monopole modes, anyway.

\footnotetext{
${ }^{4} T$ is the integration time, assumed very large. The peaks in the $\delta$ functions diverge like $T / \pi$.
} 
TABLE I. First few monopole (left) and quadrupole (right) sphere parameters, for a $\sigma=0.33$ material. First and second columns on either side of the central line number the modes and give the corresponding eigenvalue; rows are intertwined in order of ascending frequency, which is proportional to $k R$ - see (B6). The third column contains the $a_{n}$ and $b_{n}$ coefficients defined in Eqs. (3.3a) and (3.3b), respectively; the fourth column displays the cross section ratios $\left(k_{10} a_{1} / k_{n 0} a_{n}\right)^{2}$ and $\left(k_{12} b_{1} / k_{n 2} b_{n}\right)^{2}$ for higher-frequency modes, respectively, taking as reference the lowest in each familiy -cf. Eqs. (4.18).

\begin{tabular}{cccc|cccc}
\hline \hline$n$ & $k_{n 0} R$ & $a_{n} / R$ & $\sigma_{10} / \sigma_{n 0}$ & $n$ & $k_{n 2} R$ & $b_{n} / R$ & $\sigma_{12} / \sigma_{n 2}$ \\
\hline & & & & 1 & 2.650 & 0.328 & 1 \\
& & & & 2 & 5.088 & 0.106 & 2.61 \\
1 & 5.432 & 0.214 & 1 & & & & \\
& & & & 3 & 8.617 & $-1.907 \times 10^{-2}$ & 27.95 \\
& & & & 4 & 10.917 & $-9.101 \times 10^{-3}$ & 76.42 \\
& 12.138 & $-3.772 \times 10^{-2}$ & 6.46 & & & & \\
& & & & 5 & 12.280 & $1.387 \times 10^{-2}$ & 25.99 \\
3 & 18.492 & $1.600 \times 10^{-2}$ & 15.49 & 6 & 15.347 & $6.879 \times 10^{-3}$ & 67.87 \\
\hline \hline
\end{tabular}

$$
\begin{aligned}
W(\omega)= & \frac{1}{2} \pi M \sum_{n=1}^{\infty} a_{n}^{2}\left|G^{(S)}(\omega)\right|^{2}\left[\delta\left(\omega-\omega_{n 0}\right)+\delta\left(\omega+\omega_{n 0}\right)\right] \\
& +\frac{1}{2} \pi M \sum_{n=1}^{\infty} b_{n}^{2}\left[\sum_{m=-2}^{2}\left|G^{(m)}(\omega)\right|^{2}\right]\left[\delta\left(\omega-\omega_{n 2}\right)+\delta\left(\omega+\omega_{n 2}\right)\right] .
\end{aligned}
$$

The energy at any one spectral frequency $\omega_{n l}$ is obtained by integration of the spectral density in a narrow interval around $\omega= \pm \omega_{n l}$ :

$$
E\left(\omega_{n l}\right)=\int_{-\omega_{n l}-\varepsilon}^{-\omega_{n l}+\varepsilon}+\int_{\omega_{n l}-\varepsilon}^{\omega_{n l}+\varepsilon} W(\omega) \frac{d \omega}{2 \pi} .
$$

In particular,

$$
\begin{aligned}
& E\left(\omega_{n 0}\right)=\frac{1}{2} M a_{n}^{2}\left|G^{(S)}\left(\omega_{n 0}\right)\right|^{2} \\
& E\left(\omega_{n 2}\right)=\frac{1}{2} M b_{n}^{2} \sum_{m=-2}^{2}\left|G^{(m)}\left(\omega_{n 2}\right)\right|^{2} .
\end{aligned}
$$

The sensitivity parameter associated with the vibrational energy of the modes is the detector's absorption cross section, defined as the energy it absorbs per unit incident GW spectral flux density or

$$
\sigma_{\mathrm{abs}}(\omega)=\frac{E(\omega)}{\Phi(\omega)}
$$

where $\Phi(\omega)$ is the number of joules per square meter and $\mathrm{Hz}$ carried by the GW at frequency $\omega$ as it passes by the antenna. Thus, for the frequencies of interest,

$$
\begin{aligned}
\sigma_{\mathrm{abs}}\left(\omega_{n 0}\right) & =\frac{1}{2} M a_{n}^{2} \frac{\left|G^{(S)}\left(\omega_{n 0}\right)\right|^{2}}{\Phi\left(\omega_{n 0}\right)} \\
\sigma_{\mathrm{abs}}\left(\omega_{n 2}\right) & =\frac{1}{2} M b_{n}^{2} \frac{\sum_{m=-2}^{2}\left|G^{(m)}\left(\omega_{n 2}\right)\right|^{2}}{\Phi\left(\omega_{n 2}\right)} .
\end{aligned}
$$

These quantities have very precise values, but such values can only be calculated on the basis of a specific underlying theory of the $G W$ physics. In the absence of such theory, neither $\Phi(\omega)$ nor $G^{(S, m)}(\omega)$ can possibly be calculated, since they are not theory-independent quantities. To date, only GR calculations have been reported in the literature $[7,9,10]$. As I will now show, even though the fractions in the RHS of (4.10) are not theory independent, some very general results can still be obtained about the sphere's cross section within the context of metric theories of the gravitational interaction. To do so, it will be necessary to go into a short digression on the general nature of weak metric GW's.

No matter which is the (metric) theory which happens to be the "correct one" to describe gravitation, it is beyond reasonable doubt that any GW's reaching the Earth ought to be very weak. The linear approximation should therefore be an extremely good one to describe the propagating field variables in the neighborhood of the detector. In such circumstances, the field equations can be derived from a Poincaré-invariant variational principle based on an action integral of the type

$$
\int \mathcal{L}\left(\psi_{A}, \psi_{A, \mu}\right) d^{4} x
$$

where the Lagrangian density $\mathcal{L}$ is a quadratic functional of the field variables $\psi_{A}(x)$ and their space-time derivatives $\psi_{A, \mu}(x)$; these variables include the metric perturbations $h_{\mu \nu}$, plus any other fields required by the specific theory under consideration, e.g., a scalar field in the the- 
ory of Brans and Dicke, etc. The requirement that $\mathcal{L}$ be quadratic ensures that the Euler-Lagrange equations of motion are linear.

The energy and momentum transported by the waves can be calculated in this formalism in terms of the components $\tau^{\mu \nu}$ of the canonical energy-momentum tensor ${ }^{5}$

$$
\tau^{\mu \nu}(\mathbf{x}, t)=\sum_{A} \frac{\partial \mathcal{L}}{\partial \psi_{A, \mu}} \psi_{A}^{, \nu}-\mathcal{L} \eta^{\mu \nu} .
$$

The flux energy density, or Poynting, vector is given by $S_{i}=c^{2} \tau^{0 i}$, i.e.,

$$
\mathbf{S}(\mathbf{x}, t)=c^{3} \sum_{\boldsymbol{A}} \frac{\partial \mathcal{L}}{\partial \dot{\psi}_{\boldsymbol{A}}} \nabla \psi_{A},
$$

where an overdot denotes $\partial / \partial t$. Any GW hitting the antenna will be seen plane, due to the enormous distance to the source. If $\mathbf{k}$ is the incidence direction (normal to the wave front), then the fields will depend on the variable $c t-\mathbf{k} \cdot \mathbf{x}$, so that the GW energy reaching the detector per unit time and area is

$$
\phi(t) \equiv \mathbf{k} \cdot \mathbf{S}(\mathbf{x}, t)=-c^{2} \sum_{A} \frac{\partial \mathcal{L}}{\partial \dot{\psi}_{A}} \dot{\psi}_{A},
$$

where $\mathbf{x}$ is the sphere's center position relative to the source, which is fixed, and so its dependence can be safely dropped in the LHS of the above expression. The important thing to note in Eq. (4.14) is that it tells us that $\phi(t)$ can be written as a quadratic form in the time derivatives of the fields $\psi_{A}$. As a consequence, the spectral density $\Phi(\omega)$, defined by

$$
\int_{-\infty}^{\infty} \phi(t) d t=\int_{0}^{\infty} \Phi(\omega) \frac{d \omega}{2 \pi}
$$

can be ascertained to factorize as

$$
\Phi(\omega)=\omega^{2} \Phi_{0}(\omega)
$$

where $\Phi_{0}(\omega)$ is again a quadratic function of the Fourier transforms $\Psi_{A}(\omega)$ of the fields $\psi_{A}$. On the other hand, the functions $G^{(S, m)}(\omega)$ in (4.10), which, it is recalled, are the Fourier transforms of $g^{(S, m)}(t)$ in $(2.20)$, contain second-order derivatives of the metric fields $h_{\mu \nu}$, and therefore of all the fields $\psi_{A}$ as a result of the theory's field equations. Since we are considering plane wave solutions to those equations, all derivatives can be reduced to time derivatives, just like in (4.14) above. We can thus write

$$
G^{(S, m)}(\omega)=-\omega^{2} \Psi^{(S, m)}(\omega),
$$

with $\Psi^{(S, m)}(\omega)$ suitable linear combinations of the $\Psi_{A}(\omega)$. Replacing the last two equations into (4.10) and manipulating dimensions expediently, we come to the remarkable result that

$$
\begin{aligned}
& \sigma_{\mathrm{abs}}\left(\omega_{n 0}\right)=K_{S}(\aleph) \frac{G M v_{t}^{2}}{c^{3}}\left(k_{n 0} a_{n}\right)^{2} \\
& \sigma_{\mathrm{abs}}\left(\omega_{n 2}\right)=K_{Q}(\aleph) \frac{G M v_{t}^{2}}{c^{3}}\left(k_{n 2} b_{n}\right)^{2}
\end{aligned}
$$

where $v_{t}^{2} \equiv(2+2 \sigma)^{-1} v_{s}^{2}, v_{s}$ being the speed of sound in the detector's material, and $\sigma$ its Poisson ratio; $G$ is the gravitational constant. What is "remarkable" about the above is that the coefficients $K_{S}(\aleph)$ and $K_{Q}(\aleph)$ are independent of frequency: They exclusively depend on the underlying gravitation theory, which I symbolically denote by $\aleph$. To see that this is the case, it is enough to consider a monochromatic incident wave: Since the coefficients $K_{S}(\aleph)$ and $K_{Q}(\aleph)$ happen to be invariant with respect to field amplitude scalings, this means they will only depend on the amplitudes' relative weights, i.e., on the field equations' specific structure.

By way of example, it is interesting to see what the results for general relativity (GR) and Brans-Dicke (BD) theory are. After somehow lengthy algebra it is found that

$$
\aleph=\mathrm{GR} \Rightarrow\left\{\begin{array}{l}
K_{S}(\aleph)=0, \\
K_{Q}(\aleph)=\frac{16 \pi^{2}}{15},
\end{array}\right.
$$

and

$$
\aleph=\mathrm{BD} \Rightarrow\left\{\begin{array}{l}
K_{S}(\aleph)=\frac{8 \pi^{2}}{9}(3+2 \Omega)^{-2} k\left[1+\frac{k \Omega}{(3+2 \Omega)^{2}}\right]^{-1}, \\
K_{Q}(\aleph)=\frac{16 \pi^{2}}{15}\left[1+\frac{1}{6}(3+2 \Omega)^{-2} k\right]\left[1+\frac{k \Omega}{(3+2 \Omega)^{2}}\right]^{-1}
\end{array}\right.
$$

\footnotetext{
${ }^{5}$ This tensor is not symmetric in general, but can be symmetrized by a standard method due to Belinfante [25,26]. For the considerations which follow in this paper it is unnecessary to go into those details, and the canonical form (4.12) will be sufficient.
} 
In the latter formulas, $\Omega$ is the usual Brans-Dicke parameter $\omega$ [27], renamed here to avoid confusion with frequency, and $k$ is a dimensionless parameter, generally of order one, depending on the source's properties [28]. As is well known, GR is obtained in the limit $\Omega \rightarrow \infty$ of $\mathrm{BD}$ [15]; the quoted results are of course in agreement with that limit.

Incidentally, an interesting consequence of the above equations is this: Though not explicitly shown in this paper (see, however, Ref. [7]), the presence of a scalar field in the theory of Brans and Dicke causes not only the monopole sphere's modes to be excited, but also the $m=0$ quadrupole ones; what we see in Eqs. (4.20) is that precisely $5 / 6$ of the total energy extracted from the scalar wave goes into the antenna's monopole modes, while there is still a remaining $1 / 6$ which is communicated to the quadrupoles, independently of the values of $\Omega$ and $k{ }^{6}$ This somehow nonintuitive result finds its explanation in the structure of the Riemann tensor in BD theory, in which the excess $R_{0 i 0 j}$ with respect to general relativity happens not to be proportional to the scalar part $E_{i j}^{(S)}$, but to a combination of $E_{i j}^{(S)}$ and $E_{i j}^{(0)}$.

Equations (4.18) show that, no matter which is the gravity theory assumed, the sphere's absorption cross sections for higher modes scale as the successive coefficients $\left(k_{n 0} a_{n}\right)^{2}$ and $\left(k_{n 2} b_{n}\right)^{2}$ for monopole and quadrupole modes, respectively. In particular, the result quoted in [10], that the cross section for the second quadrupole mode is 2.61 times less than that for the first, assuming GR, is in fact valid, as we now see, independently of which is the (metric) theory of gravity actually governing $G W$ physics. The fourth column in Table I displays these scaling properties. It is seen that the drop in cross section from the first to the second monopole mode is as high as 6.46. It should, however, be stressed that the frequency of such mode would be over $4 \mathrm{kHz}$ for a (likely) sphere whose fundamental quadrupole frequency be $900 \mathrm{~Hz}$ [10]. Note finally the asymptotic cross section drop as $n^{-2}$ for large $n-$ cf. Eq. (4.4) and the ensuing paragraph.

\section{CONCLUSION}

The main purpose of this paper has been to set up a sound mathematical formalism to tackle with as much generality as possible any questions related to the interaction between a resonant antenna and a weak incoming GW, with much special emphasis on the homogeneous sphere. New results have been found along this line, such as the scaling properties of cross sections for higher-frequency modes or the sensitivity of the antenna to arbitrary metric GW's; also, new ideas have been put

\footnotetext{
${ }^{6}$ Note, however, that since monopole and quadrupole detector modes occur at different frequencies, this particular distribution of energy may not be seen if the sphere's vibrations are monitored at a single resonance.
}

forward regarding the direction deconvolution problem within the context of an arbitrary metric theory of GW physics. Less spectacularly, the full machinery has also been applied to produce independent checks of previously published results.

The whole investigation reported herein has been developed with no a priori assumptions about any specific (metric) theory of the GW's, and is therefore very general. "Too general solutions" are often impractical in science; here, however, the "very general" appears to be rather "cheap," as seen in the results expressed by the equations of Sec. III above. An immediate consequence is that solid elastic detectors of GW's (and, in particular, spheres) offer, as a matter of principle, the possibility of probing any given theory of GW physics with just as much effort as it would take, e.g., to probe general relativity: The vector transfer function of Sec. IV supplies the requisite theoretical vehicle for the purpose.

An important question, however, has not been considered in this paper. This is the transducer problem: The sphere's oscillations can only be revealed to the observer by means of suitable (usually electromechanical) transducers. These devices, however, are not neutral; i.e., they couple to the antenna's motions, thereby excercising a back action on it which must be taken into consideration if one is to correctly interpret the system's readout. Prelinninary studies and proposals have already been published [8], but further work is clearly needed for a more thorough understanding of the problems involved.

Progress in this direction is currently being made, which I expect to report on shortly. The formalism developed in this paper provides basic support to that further work.

\section{ACKNOWLEDGMENTS}

It is a pleasure for me to thank Eugenio Coccia for his critical reading and comments on the manuscript, and to J.M.M. Senovilla for having devoted a part of his time to enlightening discussions with me on the geometrical nature and properties of metric GW's. I also want to express gratitude to M. Montero and J.A. Ortega for their assistance during the first stages of this work. I have received support from the Spanish Ministry of Education through Contract NO. PB93-1050.

\section{APPENDIX A}

Let $\mathbf{e}_{x}, \mathbf{e}_{y}, \mathbf{e}_{z}$ be three orthonormal Cartesian vectors defining the sphere's laboratory reference frame. We define the equivalent triad

$$
\mathbf{e}^{(0)}=\mathbf{e}_{z}, \quad \mathbf{e}^{( \pm 1)}=\frac{1}{\sqrt{2}}\left(\mathbf{e}_{x} \pm i \mathbf{e}_{y}\right),
$$

having the properties

$$
\mathbf{e}^{*\left(m^{\prime}\right)} \cdot \mathbf{e}^{(m)}=\delta_{m^{\prime} m}, \quad m, m^{\prime}=-1,0,1 .
$$


We say that the vectors (A1) are the natural basis for the $l=1$ irreducible representation of the rotation group; they behave under arbitrary rotations precisely like the spherical harmonics $Y_{1 m}(\theta, \varphi)$. In particular, if a rotation of angle $\alpha$ around the $z$ axis is applied to the original frame, then

$$
\mathbf{e}^{( \pm 1)} \rightarrow \exp ( \pm i \alpha) \mathbf{e}^{( \pm 1)}, \quad \mathbf{e}^{(0)} \rightarrow \mathbf{e}^{(0)} .
$$

Higher-rank tensors have specific multipole characteristics depending on the number of tensor indices, and the above basis lends itself to reveal those characteristics, too. For example, the five-dimensional linear space of traceless symmetric tensors supports the $l=2$ irreducible representation of the rotation group, while a tensor's trace is an invariant. A general symmetric tensor can be expressed as an "orthogonal" sum of a traceless symmetric tensor and a multiple of the unit tensor. A convenient basis to expand any such tensor is

$$
\begin{aligned}
& \mathbf{e}^{(1)} \otimes \mathbf{e}^{(1)}, \quad \mathbf{e}^{(-1)} \otimes \mathbf{e}^{(-1)}, \\
& \mathbf{e}^{(0)} \otimes \mathbf{e}^{(1)}+\mathbf{e}^{(1)} \otimes \mathbf{e}^{(0)}, \quad \mathbf{e}^{(0)} \otimes \mathbf{e}^{(-1)}+\mathbf{e}^{(-1)} \otimes \mathbf{e}^{(0)}
\end{aligned}
$$

$\mathbf{e}^{(1)} \otimes \mathbf{e}^{(-1)}+\mathbf{e}^{(-1)} \otimes \mathbf{e}^{(1)}-2 \mathbf{e}^{(0)} \otimes \mathbf{e}^{(0)}$,

$$
\mathbf{e}^{(1)} \otimes \mathbf{e}^{(-1)}+\mathbf{e}^{(-1)} \otimes \mathbf{e}^{(1)}+\mathbf{e}^{(0)} \otimes \mathbf{e}^{(0)}
$$

The elements (A4a) get multiplied by $e^{ \pm 2 i \alpha}$ in a rotation of angle $\alpha$ around the $z$ axis, respectively, the (A4b) by $e^{ \pm i \alpha}$, and (A4c) and (A4d) are invariant, as is readily seen. These properties define the "spin characteristics" of the corresponding tensors. Also, the five elements (A4a)(A4c) are traceless tensors, while (A4d) is the unit tensor. Any symmetric tensor can be expressed as a linear combination of the six equations of (A4), and the respective coefficients carry information about the weights of the different monopole and quadrupole components of the tensor.

Equations (2.16) in the text are the matrix representation of the above tensors in the Cartesian basis, $\mathbf{e}_{x}, \mathbf{e}_{y}, \mathbf{e}_{z}$, except that they are multiplied by suitable coefficients to ensure that the conditions

$$
E_{i j}^{(S)} n_{i} n_{j}=Y_{00}(\theta, \varphi) \quad, \quad E_{i j}^{(m)} n_{i} n_{j}=Y_{2 m}(\theta, \varphi)
$$

where $\mathbf{n} \equiv \mathbf{x} /|\mathbf{x}|$ is the radial unit vector, hold. They are arbitrary, but expedient for the calculations in this paper. The following orthogonality relations can be easily established:

$$
\begin{gathered}
E_{i j}^{*\left(m^{\prime}\right)} E_{i j}^{(m)}=\frac{15}{8 \pi} \delta_{m^{\prime} m}, E_{i j}^{(S)} E_{i j}^{(m)}=0 \\
E_{i j}^{(S)} E_{i j}^{(S)}=\frac{3}{4 \pi}
\end{gathered}
$$

with the indices $m, m^{\prime}$ running from -2 to 2 , and with an understood sum over the repeated $i$ and $j$. It is also easy to prove the closure properties

$$
E_{i j}^{(S)} E_{k l}^{(S)}+\frac{2}{5} \sum_{m=-2}^{2} E_{i j}^{*(m)} E_{k l}^{(m)}=\frac{3}{8 \pi}\left(\delta_{i k} \delta_{j l}+\delta_{i l} \delta_{j k}\right)
$$

Equations (A6) and (A7) constitute the completeness equations of the $E$-matrix basis of Euclidean symmetric tensors.

\section{APPENDIX B}

This appendix is intended to give a rather complete summary of the frequency spectrum and eigenmodes of a uniform elastic sphere. Although this is a classical problem in elasticity theory [29], some of the results which follow have never been published so far. Also, its scope is to serve as reference for notation, etc., in future work.

The uniform ${ }^{7}$ elastic sphere's normal modes are obtained as the solutions to the eigenvalue equation

$$
\mu \nabla^{2} \mathbf{u}+(\lambda+\mu) \nabla(\nabla \cdot \mathbf{u})=-\omega^{2} \rho \mathbf{u}
$$

with the boundary conditions that its surface be free of any tensions and/or tractions; this is expressed by the equations [11]

$$
\sigma_{i j} n_{j}=0 \text { at } r=R
$$

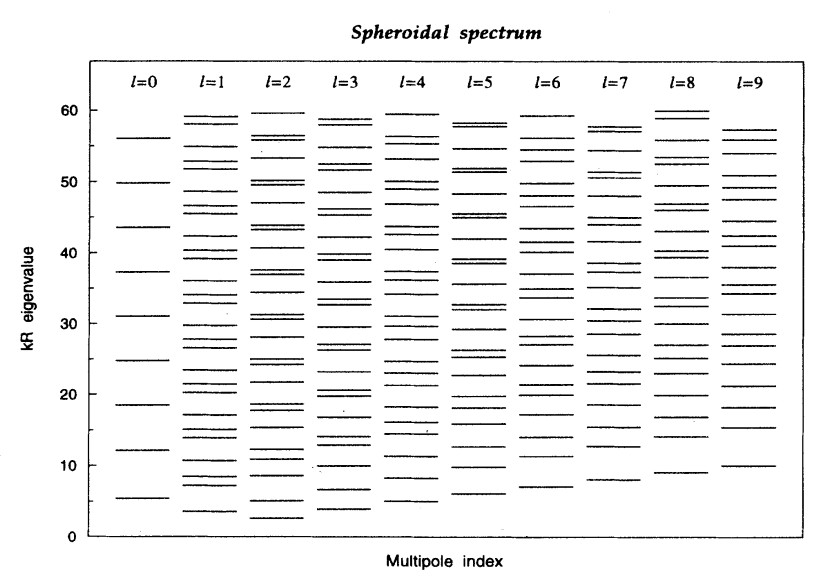

FIG. 3. The homogeneous sphere spheroidal eigenvalues for a few multipole families. Only the $l=0$ and $l=2$ families couple to metric GW's, and so the rest are given for completeness and nondirect GW uses. Note that there are fewer monopole than any other $l$-pole modes. The lowest frequency is the first quadrupole. The diagram corresponds to a sphere with Poisson ratio $\sigma=0.33$. Frequencies can be obtained from the plotted values through Eq. (B6) for any specific case.

\footnotetext{
${ }^{7} \mathrm{By}$ uniform I mean its density $\rho$ is constant throughout the solid in the unperturbed state.
} 


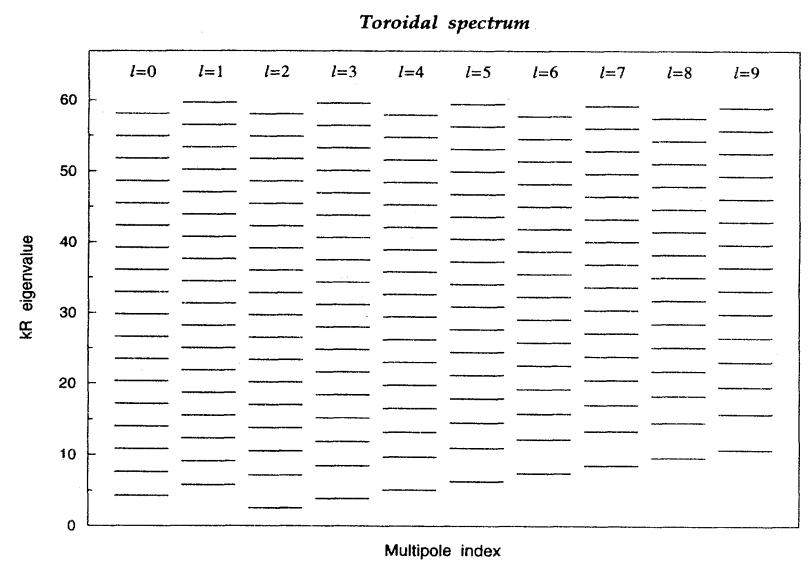

FIG. 4. The homogeneous sphere toroidal eigenvalues. None of these couple to GW's, but knowledge of them can be useful for vetoing purposes. These eigenvalues are independent of the material's Poisson ratio. To obtain actual frequencies from plotted values, use (B6). The lowest toroidal eigenvalue is $k R=2.5011$, with $l=2$, and happens to be the absolute minimum sphere's eigenvalue. Compared to the spheroidal $k R=2.6497$, also with $l=2$, its frequency is $5.61 \%$ smaller.

where $R$ is the sphere's radius, $\mathbf{n}$ the outward normal, and $\sigma_{i j}$ the stress tensor,

$$
\sigma_{i j}=\lambda u_{k k} \delta_{i j}+2 \mu u_{i j}
$$

with $u_{i j} \equiv \frac{1}{2}\left(u_{i, j}+u_{j, i}\right)$, the strain tensor, and $\lambda, \mu$ the Lamé coefficients [11]. $\mathbf{u}(\mathbf{x})$ can be expressed as a combination of an irrotational vector and two divergence free vectors:

$$
\mathbf{u}(\mathbf{x})=C_{0} \nabla \phi(\mathbf{x})+i C_{1} \mathbf{L} \psi(\mathbf{x})+i C_{2} \nabla \times \mathbf{L} \psi(\mathbf{x})
$$

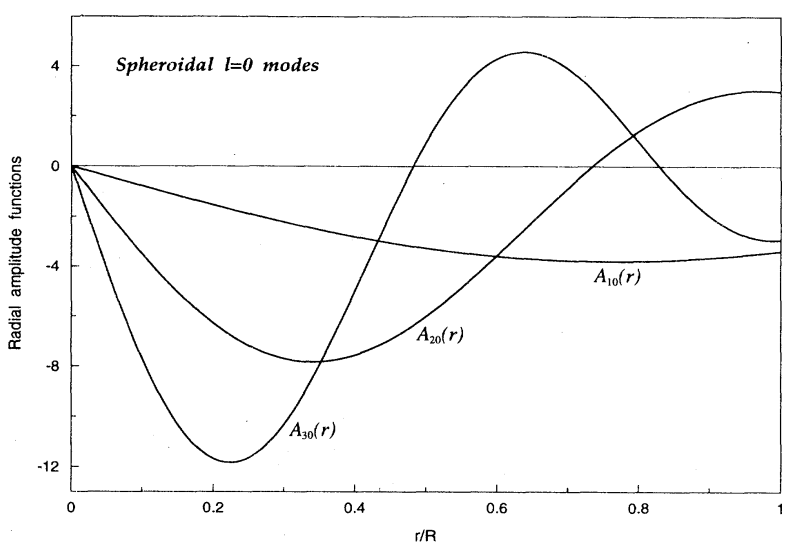

FIG. 5. First three spheroidal monopole radial functions $A_{n 0}(r)(n=1,2,3)$, Eq. (B14a).

where $C_{0}, C_{1}, C_{2}$ are constants, and $\mathbf{L} \equiv-i \mathbf{x} \times \nabla$ is the "angular momentum" operator. If (B4) is to satisfy (B1), then the scalar functions $\phi, \psi$ must be

$$
\phi(\mathbf{x})=j_{l}(q r) Y_{l m}(\theta, \varphi), \quad \psi(\mathbf{x})=j_{l}(k r) Y_{l m}(\theta, \varphi)
$$

in order to ensure regularity at the center $r=0$. Here, $j_{l}$ is a spherical Bessel function (see [30] for general conventions on these functions) and $Y_{l m}$ a spherical harmonic [16]. Finally,

$$
q^{2} \equiv \frac{\rho \omega^{2}}{\lambda+2 \mu}, \quad k^{2} \equiv \frac{\rho \omega^{2}}{\mu} .
$$

The boundary conditions (B2) are, after lengthy algebra, expressed as the following system of linear equations for $C_{0}, C_{1}, C_{2}$ :

$$
\begin{array}{r}
{\left[\beta_{2}(q R)-\frac{\lambda}{2 \mu} q^{2} R^{2} \beta_{0}(q R)\right] C_{0}-l(l+1) \beta_{1}(k R) C_{2}=0} \\
\beta_{1}(k R) C_{1}=0 \\
\beta_{1}(q R) C_{0}-\left[\frac{1}{2} \beta_{2}(k R)+\left\{\frac{l(l+1)}{2}-1\right\} \beta_{0}(k R)\right] C_{2}=0
\end{array}
$$

where

$$
\beta_{0}(z) \equiv \frac{j_{l}(z)}{z^{2}}, \quad \beta_{1}(z) \equiv \frac{d}{d z}\left[\frac{j_{l}(z)}{z}\right], \quad \beta_{2}(z) \equiv \frac{d^{2}}{d z^{2}}\left[j_{l}(z)\right] .
$$

There are clearly two families of solutions to (B7).

(i) Toroidal modes. These are characterized by

$$
\beta_{1}(k R)=0, \quad C_{0}=C_{2}=0 .
$$

The frequencies of these modes are independent of $\lambda$, and thence independent of the material's Poisson ratio. Their amplitudes are

$$
\mathbf{u}_{n l m}^{T}(\mathbf{x})=T_{n l}(r) i \mathbf{L} Y_{l m}(\theta, \varphi)
$$


with

$$
T_{n l}(r)=C_{1}(n, l) j_{l}\left(k_{n l} r\right)
$$

and $C_{1}(n, l)$ a dimensionless normalization constant determined by the general formula $(2.8) ; k_{n l} R$ is the $n$th root of the first equation (B9) for a given $l$.

(ii) Spheroidal modes. These correspond to

$$
\operatorname{det}\left(\begin{array}{cc}
\beta_{2}(q R)-\frac{\lambda}{2 \mu} q^{2} R^{2} \beta_{0}(q R) & l(l+1) \beta_{1}(k R) \\
\beta_{1}(q R) & \frac{1}{2} \beta_{2}(k R)+\left\{\frac{l(l+1)}{2}-1\right\} \beta_{0}(k R)
\end{array}\right)=0
$$

and $C_{1}=0$. The frequencies of these modes $d o$ depend on the Poisson ratio, and their amplitudes are

$$
\mathbf{u}_{n l m}^{P}(\mathbf{x})=A_{n l}(r) Y_{l m}(\theta, \varphi) \mathbf{n}-B_{n l}(r) i \mathbf{n} \times \mathbf{L} Y_{l m}(\theta, \varphi),
$$

where $A_{n l}(r)$ and $B_{n l}(r)$ have the somewhat complex form,

$$
\begin{aligned}
& A_{n l}(r)=C(n, l)\left[\beta_{3}\left(k_{n l} R\right) j_{l}^{\prime}\left(q_{n l} r\right)-l(l+1) \frac{q_{n l}}{k_{n l}} \beta_{1}\left(q_{n l} R\right) \frac{j_{l}\left(k_{n l} r\right)}{k_{n l} r}\right], \\
& B_{n l}(r)=C(n, l)\left[\beta_{3}\left(k_{n l} R\right) \frac{j_{l}\left(q_{n l} r\right)}{q_{n l} r}-\frac{q_{n l}}{k_{n l}} \beta_{1}\left(q_{n l} R\right) \frac{\left\{k_{n l} r j_{l}\left(k_{n l} r\right)\right\}^{\prime}}{k_{n l} r}\right],
\end{aligned}
$$

with primes denoting derivatives with respect to implied (dimensionless) arguments,

$$
\beta_{3}(z) \equiv \frac{1}{2} \beta_{2}(z)+\left\{\frac{l(l+1)}{2}-1\right\} \beta_{0}(z)
$$

and $C(n, l)$ a new normalization constant. It is understood that $q_{n l}$ and $k_{n l}$ are obtained after the (trascen-

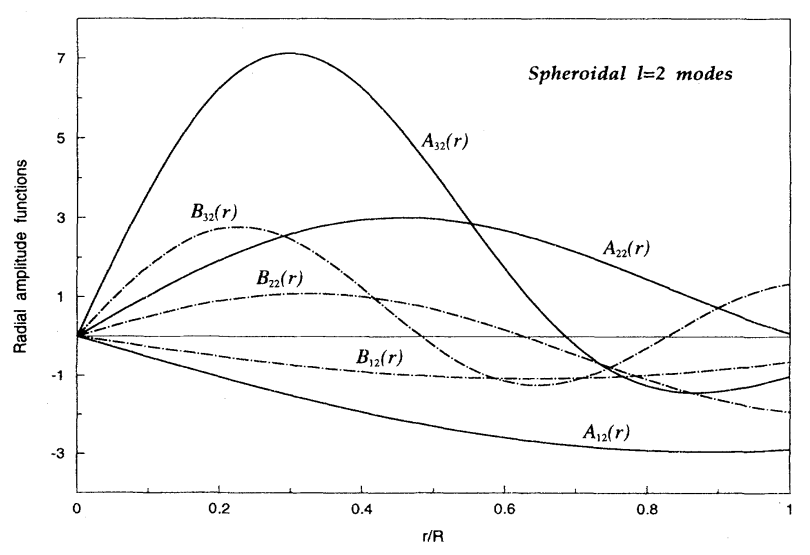

FIG. 6. First three spheroidal quadrupole radial functions $A_{n 2}(r)$ (solid line) and $B_{n 2}(r)$ (dashed line) $(n=1,2,3)$, Eqs. (B14). dental) equation (B12) has been solved for $\omega-$ cf. Eq. (B6).

In Figs. 3 and 4 a symbolic line diagram of the two families of frequencies of the sphere's spectrum is presented. Spheroidal eigenvalues have been plotted for the usual Poisson ratio $\sigma=0.33$. Although only the $l=0$ and $l=2$ spheroidal series couple to GW tidal forces, the plots include other eigenvalues, as they can be useful both in bench experiments [cf. Eq. (2.12) above] and for vetoing

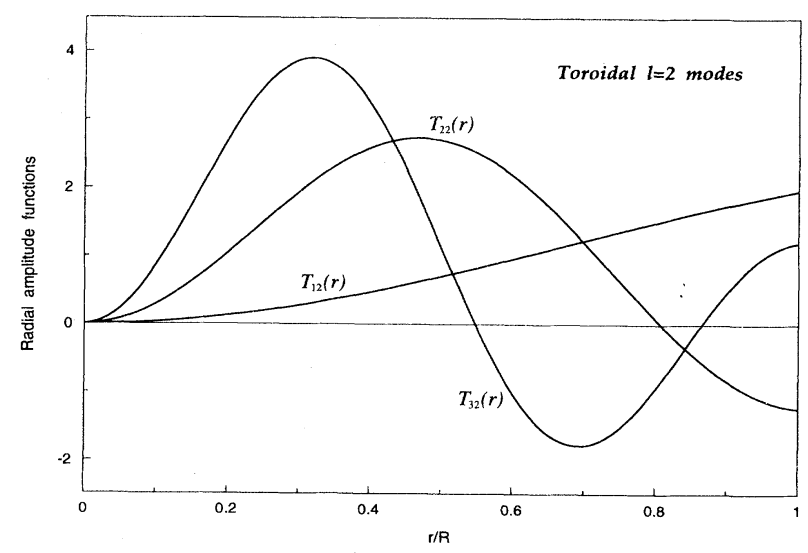

FIG. 7. First three toroidal quadrupole radial functions $T_{n 2}(r)(n=1,2,3)$, Eq. (B11). A common feature to these radial functions (also in the two previous figures) is that they present a nodal point at the origin $(r=0)$, while the sphere's surface $(r / R=1)$ has a nonzero amplitude value, which is largest (in absolute value) for the lowest $n$ in each group. 
purposes in a spherical antenna.

Figures 5-7 contain plots of the first three monopole and quadrupole functions $T_{n l}(r), A_{n l}(r)$, and $B_{n l}(r)$, always for $\sigma=0.33$. $T_{n 0}(r)$ and $B_{n 0}(r)$ have, however, been omitted; this is because they are multiplied by an identically zero angular coefficient in the amplitude formulas (B10) and (B13). Indeed, monopole vibrations are spherically symmetric, i.e., purely radial.
[1] See, e.g., O.W. Hamilton, in General Relativity and Gravitation 1992, Proceedings of the 13th Conference Córdoba, Argentina, 1992, edited by R.J. Gleiser, C.N. Kozameh, and O.M. Moreschi (IOP, London, 1993); also, O.W. Hamilton, in Proceedings of the First Edoardo Amaldi Conference, Frascati, Italy, edited by E. Coccia, G. Pizzella, and F. Ronga (World Scientific, Singapore, 1995).

[2] P. Astone et al., Phys. Rev. D 47, 362 (1993).

[3] P. Astone et al., Europhys. Lett. 16, 231 (1991).

[4] Eugenio Coccia (personal communication).

[5] R. Forward, Gen. Relativ. Gravit. 2, 149 (1971).

[6] N. Ashby and J. Dreitlein, Phys. Rev. D 12, 336 (1975).

[7] R.V. Wagoner and H.J. Paik, in Experimental Gravitation, Proceedings of the Pavia International Symposium (Academia Nazionale dei Lincei, Roma, Italy, 1977), pp. 257-266.

[8] W. Johnson and S.M. Merkowitz, Phys. Rev. Lett. 70, 2367 (1993).

[9] P.F. Michelson and C.Z. Zhou, Phys. Rev. D 51, 2517 (1995).

[10] E. Coccia, J.A. Lobo, and J.A. Ortega (unpublished).

[11] L.D. Landau and E.M. Lifshitz, Theory of Elasticity (Pergamon, New York, 1970).

[12] Myint-U Tym, Partial Differential Equations for Scientists and Engineers (North-Holland, Amsterdam, 1987).

[13] Warren Johnson (private communication).

[14] C.W. Misner, K.S. Thorne, and J.A. Wheeler, Gravitation (Freeman, San Francisco, 1973).

[15] S. Weinberg, Gravitation and Cosmology (Wiley, New York, 1972).
[16] A.R. Edmonds, Angular Momentum in Quantum Mechanics (Princeton University Press, Princeton, 1960).

[17] S.N. Rasband, J. Acoust. Soc. Am. 57, 899 (1975).

[18] R.G. Hier and S.N. Rasband, Astrophys. J. 195, 507 (1975).

[19] M. Bassan, C. Cosmelli, S. Frasca, M.A. Papa, P. Puppo, P. Rapagnani, and F. Ricci, "High Frequency Gravitational Array, La Sapienza" Roma report, 1994 (unpublished).

[20] J.A. Lobo and J.A. Ortega, in Proceedings of the First Edoardo Amaldi Conference [1].

[21] I take this denomination from Ref. [9] above. It is, apparently, less common in the classical literature than the term toroidal applied to the other modes.

[22] D.M. Eardley, D.L. Lee, and A.P. Lightman, Phys. Rev. D 8, 3308 (1973).

[23] P. Astone, J.A. Lobo, and B.F. Schutz, Class. Quantum Grav. 11, 2093 (1994).

[24] C.W. Helstrom, Statistical Theory of Signal Detection (Pergamon, New York, 1968).

[25] L.D. Landau and E.M. Lifshitz, The Classical Theory of Fields (Pergamon, New York, 1985).

[26] A.O. Barut, Electrodynamics and Classical Theory of Fields and Particles (Dover, New York, 1980).

[27] C. Brans and R.H. Dicke, Phys. Rev. 124, 925 (1961).

[28] J.A. Lobo (unpublished).

[29] A.E.H. Love, A Treatise on the Mathematical Theory of Elasticity (Dover, New York, 1944).

[30] Handbook of Mathematical Functions, edited by M. Abramowitz and I.A. Stegun (Dover, New York, 1972). 